Ingeniería

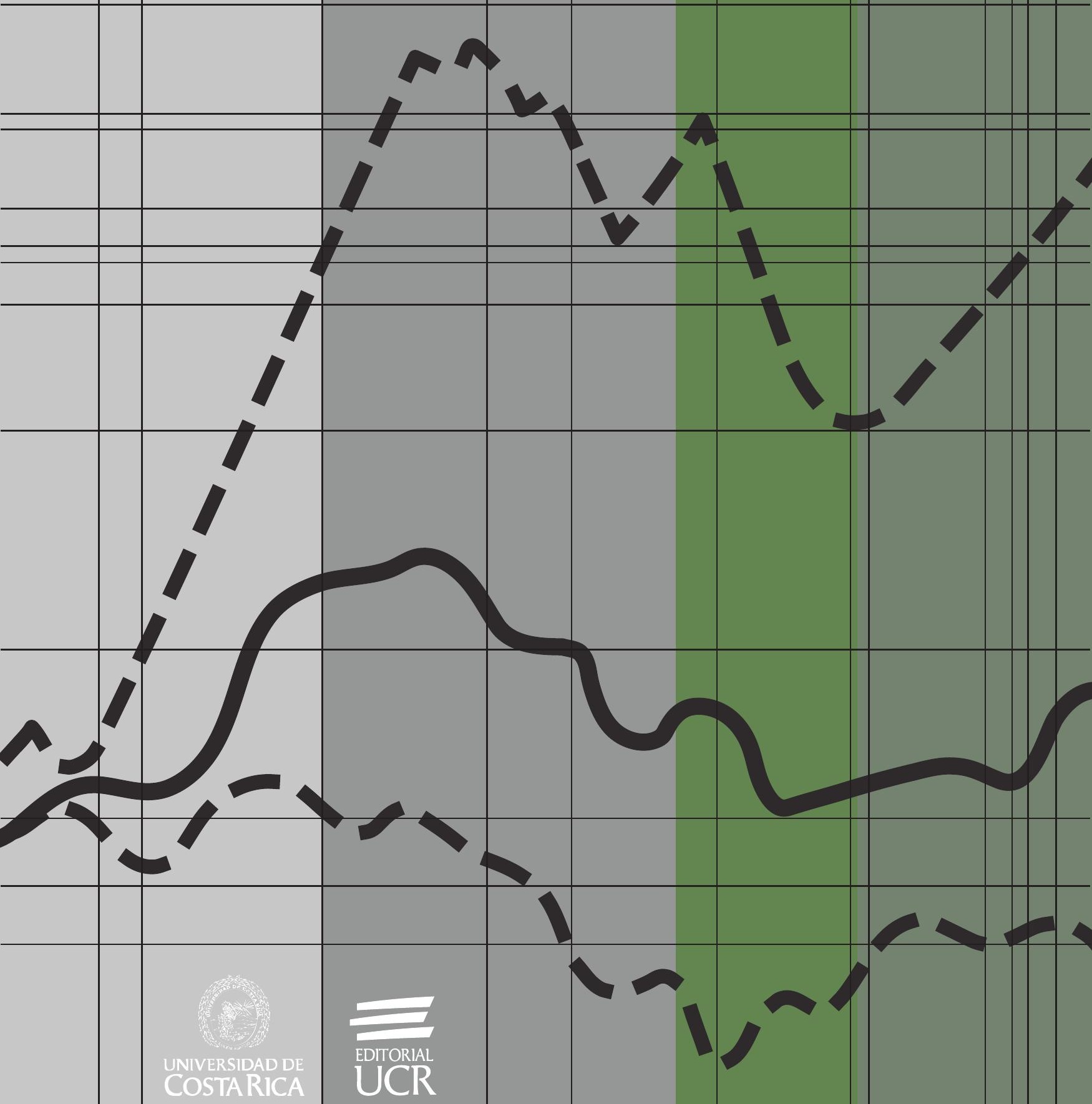




\title{
Validación de un modelo de gestión empresarial para pequeñas y medianas empresas (PYMES) basado en dinámica de sistemas
}

\author{
Validation of Business Management Model for Small \\ and Medium Sized Enterprises (SMEs) Based on System \\ Dynamics \\ Hernan Washington Samaniego Guevara \\ Universidad Politécnica Salesiana \\ Quito-Ecuador \\ hernn_wsg@yahoo.com
}

Recibido: 25 de octubre 2017

Aceptado: 10 de abril 2018

\begin{abstract}
Resumen
El propósito de este trabajo investigativo es demostrar la validación de un modelo de gestión empresarial diseñado acorde a la metodología de la dinámica de sistemas, el cual fue aplicado exclusiva y específicamente a las pequeñas y medianas empresas (PYMES). La validación del modelo posibilita tener la seguridad de que está en concordancia con las necesidades organizacionales de este tipo de empresas.

Para la validación del modelo se utiliza la técnica de recopilación de datos, utilizando para esto información seleccionada y obtenida durante un lapso de tiempo específico, analizando que dicha información sea relevante, confiable y correcta. Se consideró que, al estudiar un determinado sistema, conviene analizar su comportamiento a partir de datos representativos de lo que se espera que sea su funcionamiento habitual y no con datos correspondientes a situaciones extremas.

La conjunción de la metodología de la dinámica de sistemas y la técnica de recopilación de datos para validar el modelo generado, posibilita efectuar simulaciones que permiten a las PYMES ratificar o rectificar las políticas de gestión administrativas que aplican al interior de sus empresas.
\end{abstract}

\section{Palabras Claves}

Modelo de gestión empresarial, validación, simulaciones, metodología, recopilación de datos

\begin{abstract}
The purpose of this research is to demonstrate the validation of a business management model designed according to the methodology of system dynamics, which is applied exclusively and specifically to small and medium enterprises (SMEs). The validation of the model makes it possible to be sure that it is in accordance with the organizational needs of this type of company.
\end{abstract}


For the validation of the model the data collection technique was applied, using information selected and obtained during a specific time period, analyzing that such information is relevant, reliable and correct; considering that, when studying a certain system, it is convenient to analyze its behavior from representative data of what is expected to be its normal operation and not with data corresponding to extreme situations.

The combination of the system dynamics methodology and the data collection technique to validate the generated model makes it possible to carry out simulations which allow SMEs to ratify or rectify the administrative management policies that they apply to their companies.

\section{Keywords}

Business management model, validation, simulations, methodology, data collection. 


\section{INTRODUCCIÓN}

Las pequeñas y medianas empresas (PYMES) son consideradas como generadoras de empleo y como elementos que posibilitan dinamizar la economía. Por lo tanto, este tipo de empresas deben buscar formas de lograr que sus actividades y productos generen valores agregados que permitan conseguir la atención de sus posibles clientes. Sin embargo, estas continúan empleando las estrategias que utilizan las grandes empresas, sin considerar que estas poseen un poder económico que les permite realizar estudios superiores, como es el caso de prepararse para un mundo globalizado (Samaniego, 2017).

En la actualidad, resulta un trabajo complicado el implementar teorías administrativas en las PYMES, por ejemplo, realizar un estudio de planificación estratégica o la elaboración de un análisis de benchmarking, que se utilizan para establecer fortalezas, debilidades, oportunidades y amenazas de una empresa en particular. Por lo general, los resultados que se obtienen permiten establecer estrategias específicas a ser implementadas, pero dichas metodologías en ocasiones no resultan ser factibles para que las PYMES las ejecuten, ya sea por falta de capital o por la carencia de poseer personal capacitado para implementar dichas metodologías.

De esta manera, las metodologías anteriormente señaladas facilitan que las pequeñas y medianas empresas puedan tener una visión actualizada del mercado en el que se desenvuelven, pero no es la única manera de realizar estrategias en una PYMES, ya que existe la posibilidad de implementar modelos de gestión empresarial aplicables a este tipo de empresas. Estos modelos pueden ser realizados en base a distintas metodologías, como los arquetipos de administración gerencial, organizacional, proyectos y aquellas configuraciones de gestión que se basan en la ejecución de simulaciones.

Igualmente, los modelos o configuraciones de gestión en base a simulaciones son posibles de realizar acorde a metodologías como la investigación de operaciones, la dinámica de sistemas, la modelación matemática, entre otras, considerando que estos modelos de gestión son útiles siempre y cuando sean realistas y simplifiquen la realidad. De esta manera, el diseñar un prototipo de gestión empresarial en base a la metodología de la dinámica de sistemas permite realizar el análisis de resultados posibles de alcanzar en un futuro si es aplicado al interior organizacional de cualquier PYMES.

Por lo tanto, y en concordancia con la aplicación de cualquier metodología para elaborar un prototipo, todo modelo realizado debe ser validado para asegurar que es confiable, no tiene errores y es aceptado por quienes lo van a utilizar (Góngora y Madrid, 2010). En tales circunstancias los modelos de gestión realizados en base a la dinámica de sistemas se deberán validar antes de su entrega o implementación en un determinado campo de trabajo.

Conforme a lo especificado, la validación es una etapa esencial que presenta dificultades. Por lo tanto, para legitimar este tipo de modelos, es posible efectuar comparaciones de las predicciones de este con observaciones provenientes del mundo real (Barrales, Peña y Fernández, 2004), ante lo cual, en muchas ocasiones el proceso de validación se ejecuta mediante la utilización de métodos estadísticos adecuados (Aguilar, 1997). 
Otros autores, en cambio, señalan que la necesidad de validar o evaluar un modelo debe centrarse en la búsqueda de un método objetivo y cuantitativo (Flavelle, 1992).

No sorprende, pues, que para efectuar una validación, los modeladores recurran a procedimientos simples a su alcance, aparentemente adecuados, incluyendo gráficos de dispersión, predicciones y observaciones, algunas veces con regresión, la cual es pensada como método objetivo y cuantitativo para medir cuán bueno es un modelo (Barrales, Peña y Fernández, 2004).

Por lo tanto, validar un modelo es necesario e importante para determinar si está realizado conforme a la necesidad que se requiere demostrar, acorde a un escenario cercano, similar o igual a la realidad que se presenta en cualquier campo de trabajo, estudio o investigación. Ante esto, es pertinente preguntar, ¿es posible validar un modelo de simulación efectuado acorde a la metodología de la dinámica de sistemas?

\section{DINÁMICA DE SISTEMAS}

La dinámica de sistemas tiene sus orígenes en las décadas de los cincuenta y sesenta, y se atribuye el inicio de esta perspectiva de modelación a Jay W. Forrester. La metodología de la dinámica de sistemas hace uso de conceptos intuitivos de representación de sistemas y puede asociarse (aunque normalmente no se hace) con las técnicas de mapas conceptuales usadas en enseñanza. Los mapas conceptuales son también grafos, pero las relaciones no se establecen con un fin operativo, sino solo con un fin organizativo. En la dinámica de sistemas, en cambio, interesa analizar cómo evoluciona un sistema en el tiempo, y la forma en la que cambia y se comportan los elementos o las variables de dicho sistema (Godoy y Bartó, 2002).

Otros estudios especifican que la dinámica de sistemas es una metodología de apoyo para pensar problemas en términos de sistemas. El objetivo es pensar acerca de sistemas complejos en los cuales hay partes, componentes, e interacciones entre ellos (Aracil y Gordillo, 1997). Para lograr lo especificado es factible utilizar un modelo computacional que evidencie las relaciones presentes entre la estructura o grafo del sistema y su comportamiento. A la vez, dichas relaciones deben proporcionar la seguridad de que conforme al diseño realizado y por lo tanto dicho diseño debe necesariamente ser validado.

Acorde a lo especificado hasta el momento, la dinámica de sistemas es una metodología que posee el potencial de complementar modelos no formales (normalmente verbales) de sistemas complejos y modelos matemáticos más abstractos (Izquierdo, Galán, Santos y Del Olmo Martínez, 2008). A menudo la representación verbal de estos sistemas complejos carece del rigor formal necesario para valorar su coherencia lógica y para generalizar situaciones a partir de ellos. Este tipo de simulación es posible realizarla utilizando técnicas de modelado computacional, en donde es viable construir sistemas que combinan la riqueza descriptiva de los modelos verbales con el rigor formal de los modelos matemáticos más abstractos.

Así, la dinámica de sistemas posibilita analizar y modelar el comportamiento de entornos complejos sistémicos, los cuales permiten la presentación de escenarios futuros optimizando recursos y tiempo a quienes lo utilizan. 


\section{VALIDACIÓN DE UN MODELO DE GESTIÓN EMPRESARIAL PARA PYMES APLICANDO DINAMICA DE SISTEMAS}

La validación científica o verificación se ejecuta en referencia explícita al sistema real que se está modelando. Validar un modelo consiste en valorar su utilidad dentro del contexto de aplicación, de acuerdo con los criterios para los cuales el modelo fue diseñado (Izquierdo, Galán, Santos y Del Olmo Martínez, 2008). Construir modelos suele ser el avance de nuestro conocimiento sobre un determinado sistema real, y esto podría valorarse estudiando la medida en que los resultados obtenidos con el modelo se ajustan a los observados en el referente empírico (Moss, Edmonds y Wallis, 1997).

Forrester (1981) señala que la validación o el grado de significancia de un modelo debería ser juzgada por su conveniencia en relación a un determinado propósito. En cambio, Iñaki (2010) señala que la validación de modelos acorde a la dinámica de sistemas consiste en cotejar la adecuación entre el comportamiento del modelo simulado y los requerimientos previstos por los usuarios finales.

Es de notar que en la dinámica de sistemas se pasa de una visión de las relaciones estructurales entre los componentes, mediatizada por conceptos, como realimentación positiva o negativa y otras construcciones complejas, a sistemas dinámicos caracterizados por sistemas de ecuaciones diferenciales cuya solución analítica o numérica proporciona el comportamiento deseado en forma de respuesta del sistema (Godoy y Bartó, 2002).

Por lo tanto, validar un modelo de simulación ejecutado mediante la dinámica de sistemas se presenta como una actividad cognitiva en sí misma, que permite conocer los sistemas del mundo real, así como entender su comportamiento en una manera en la que un enfoque analítico puro (en sentido matemático) no permitiría. En otras palabras, es reflejar la realidad existente de un escenario que ha sido estudiado y del cual se espera alcanzar resultados antes que estos se presenten en el entorno real del sistema sujeto a estudio.

Tal es el caso que se presenta en este trabajo, donde se expone la validación de un modelo de gestión empresarial diseñado a través de una metodología de investigación mixta, con la intervención de elementos cualitativos y cuantitativos para la determinación de las variables principales que formaron parte de su contexto. Su aplicación al entorno real empresarial se realiza en las pequeñas y medianas empresas de Quito-Ecuador. El diseño del modelo de simulación en gestión empresarial, basado en dinámica de sistemas, ha sido publicado en la revista científica Dyna Management en el mes de mayo del año 2017 (Samaniego y Pascual, 2017).

Dicho modelo consta básicamente de tres partes estructurales conformadas por las variables principales que, como se menciona anteriormente, fueron determinadas a través de los elementos cualitativos y cuantitativos del estudio, para finalmente ser utilizadas como variables de niveles, flujos y variables auxiliares. La primera parte incluye a las variables clientes potenciales y clientes de productos o servicios como variables de niveles; y a los nuevos clientes y la vinculación existente entre estos, como variables de flujos. La segunda parte del modelo está conformado por variables que describen aspectos internos de las empresas, elementos necesarios para el desarrollo de las actividades 
normales de una PYMES, como bodegas, personal que labora, equipos de transporte, materia prima, capacidad de atender a clientes, calidad de servicio, recursos, entre otros, siendo consideradas como variables auxiliares. Finalmente, hay una tercera parte, en la cual se toman en cuenta las variables relativas a pedidos, ingresos y costos, consideradas también como variables auxiliares y que regularmente las PYMES incurren para el desenvolvimiento de sus actividades (Samaniego y Pascal, 2017).

Por lo tanto, al obtener el modelo mencionado en las anteriores líneas, este obligatoriamente debía ser validado para su aceptación. Dicha verificación podría haber sido efectuada de diversas maneras. La primera, utilizando criterios de correspondencia entre estructuras, comportamientos y fenómenos, los cuales básicamente tratan de validar un modelo de dinámica de sistemas mediante el análisis de cada elemento con su contraparte del mundo real, considerando para ello datos históricos en un determinado tiempo del pasado traídos al presente y futuro, complementando para esto que el modelo es un fenómeno a ser observado permanente o excepcionalmente en relación al mundo real.

Otra forma posible de validar un modelo, es a través de criterios informales como: entrevistas, panel de expertos y utilizando el criterio del test de Turing. Este último, es el que más aplicabilidad tiene para demostrar la validez de un modelo, y básicamente consiste en enfrentar a los usuarios a ciegas con el sistema o con los expertos sin que puedan distinguirlos, y que den su opinión referente a su aceptabilidad o no. Al utilizar la técnica del panel de expertos y en concordancia con las ideas de Putnam (1972), estos logran ponerse de acuerdo con bastante facilidad ya que a su criterio se pueden presentar sesgos positivos y negativos de los sistemas de simulación (Godoy y Bartó, 2002). Para evitar dichos sesgos, es posible incorporar expertos que no intervinieron desde el inicio del proceso, o a su vez, usuarios que no hayan participado del desarrollo del modelo y puedan tener una actitud menos comprometida.

Es de considerar que validar un modelo acorde a los criterios informales posibilita que estos presenten un porcentaje de fallo, por cuanto los expertos pueden fracasar en el momento de acordar criterios de validez, y para el caso de sistemas muy complejos, el producto cartesiano de todas las alternativas del modelo puede hacerlo intratable en forma completa, lo cual daría como resultado una validación del modelo sin el convencimiento total del modelador del sistema.

Finalmente, es posible validar un modelo mediante la recopilación de datos. Para aplicar esta técnica y comprobar la validez de este, es necesario recopilar información pertinente de manera precisa, considerando aquella que sea relevante, confiable y correcta. Por ejemplo, si se va a estudiar un determinado sistema conviene analizar su comportamiento a partir de datos representativos de lo que se espera que sea su régimen de funcionamiento habitual y no con datos correspondientes a situaciones extremas. Esta manera de validación se ejecuta mediante la utilización de sistemas informáticos.

Por lo expuesto en las anteriores líneas, el método de recopilación de datos fue la técnica utilizada para la validación del modelo de gestión empresarial, que fue posible por las facilidades organizacionales que dos PYMES, presentes en este campo empresarial, proporcionaron para la recopilación de la información durante un lapso de 10 meses. Con los datos recolectados, y utilizando el sistema informático Vensim, fue 
posible validar el modelo diseñado, por cuanto al obtener resultados futuros mediante la realización de simulaciones es posible analizar los rendimientos que estas empresas podrían obtener, evidenciando que el modelo de gestión empresarial es factible de aplicar sin importar si las PYMES se dedican a generar bienes o servicios.

\subsection{Validación del modelo de gestión empresarial}

Al aplicar el modelo de gestión empresarial mediante la metodología de recopilación de datos o información, conforme a lo señalado en el inciso anterior, fue necesario contactar con dos empresas. La primera PYMES labora en el campo de la jardinería y se caracteriza por proveer un servicio de generación y mantenimiento de jardines, diseño de áreas verdes y demás trabajos relacionados con este giro de negocio. Esta empresa posee clientes de instalaciones, clientes de mantenimiento y clientes potenciales (variables de niveles).

Por otra parte, la segunda empresa comercializa máquinas específicas denominadas Vending Básica y Vending Completa (variables de niveles), las cuales son pedidas por clientes que venden productos relacionados a comida rápida y bebidas. En tal virtud, la PYMES negocia o entrega estas máquinas complementando sus actividades con la presencia de clientes potenciales para comprar sus productos. Las dos empresas posibilitaron realizar el seguimiento de sus actividades por un lapso de 10 meses. De esta manera, en la figura 1 se representa el modelo de gestión empresarial generado como resultado de la aplicación en la empresa de servicios (a), en la cual es factible apreciar las variables particulares de dicho modelo resaltadas en color rojo. Mientras que, para la PYMES de producción (b), es posible apreciar las variables características del modelo resaltadas en color azul.

Igualmente, es importante señalar que, tanto en la empresa de servicios (a) como de producción (b), las variables que aplican para las dos empresas se encuentran detalladas en color negro, con lo cual el modelo diseñado denota y asegura su aplicabilidad en las pequeñas y medianas empresas.

Conforme a los modelos detallados en la figura 1, y acorde a la técnica de validación utilizada, es posible efectuar las simulaciones que las PYMES deseen. Puntualmente, en estas dos empresas dichas simulaciones fueron efectuadas a una proyección de 120 meses, por cuanto dicho tiempo permite analizar los resultados que se podrían alcanzar a corto, mediano y largo plazo. Posibilitando en su momento modificar políticas de gestión si el caso así lo amerita.

Es importante aclarar que las simulaciones realizadas para validar el modelo son factibles de ejecutar utilizando cualquier variable de nivel, flujo y auxiliar, o también conjugando estas, lo que permite obtener resultados en conjunto visualizando de manera objetiva cómo estas interactúan entre sí. 


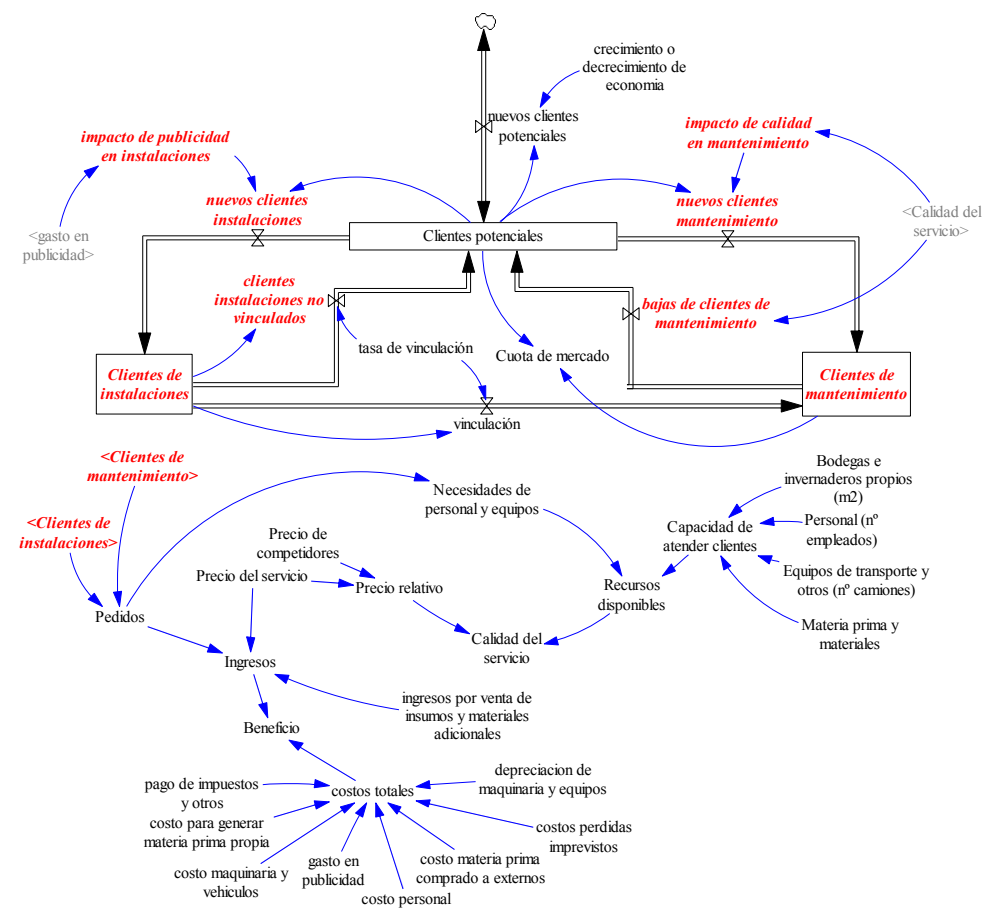

a)

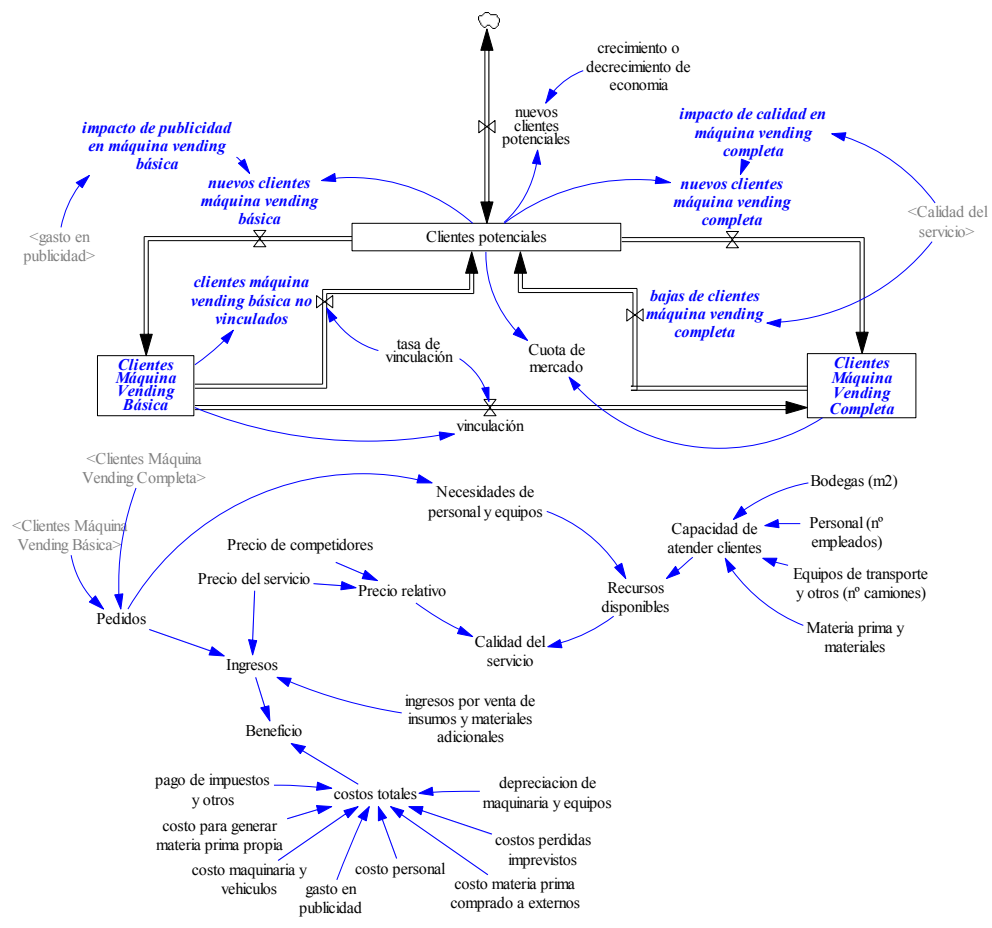

b)

Figura 1. Variables características del modelo de gestión empresarial aplicado a una PYMES de servicios (a) y a una PYMES de productos (b) 


\section{Clientes en relación a ingresos y beneficios}

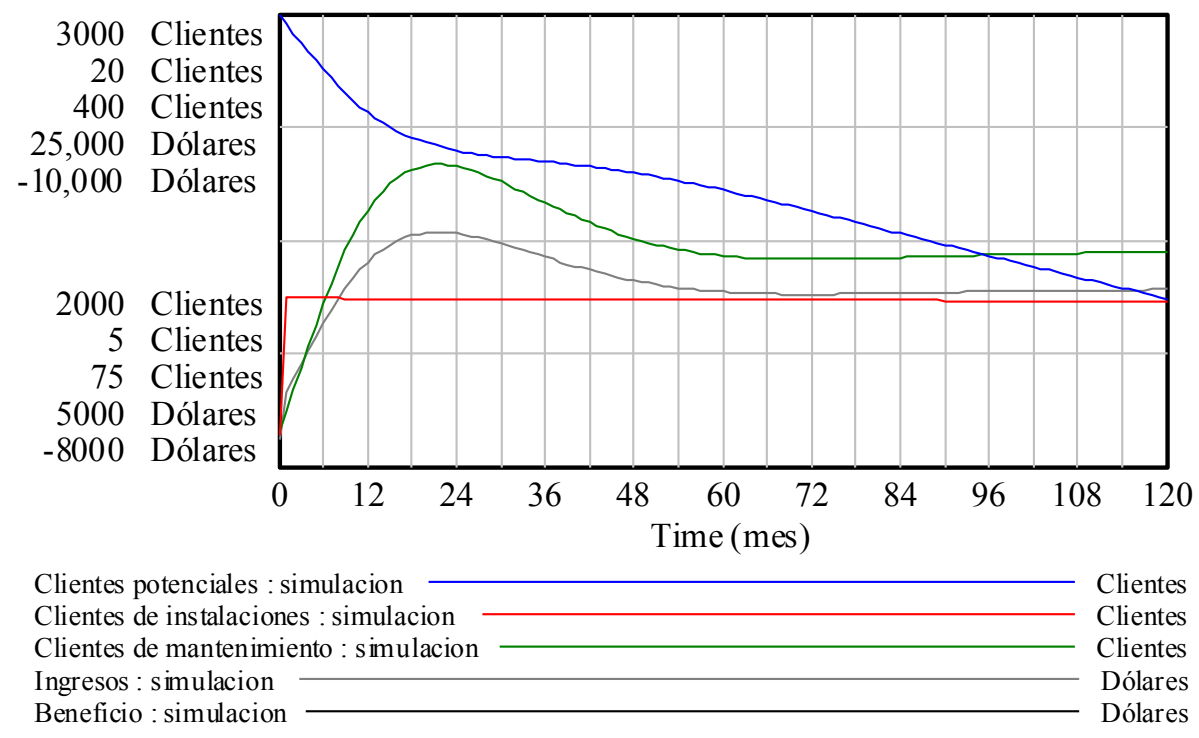

Figura 2. Simulación con base en variables clientes potenciales, clientes de instalaciones, clientes de mantenimiento, ingresos y beneficio de la PYMES de servicios (figura 1a)

Al analizar la figura 2, producto de realizar una simulación en el modelo aplicado a la empresa de servicios, es posible observar los resultados alcanzados en función de clientes, ingresos y beneficio que la PYMES obtendría en el lapso de tiempo simulado. Específicamente en esta figura, se observa que los clientes potenciales en el mes cero están ubicados en una cantidad de 3000 personas, mientras que, con el transcurso del tiempo, esta tendencia disminuye hasta ubicarse en un nivel de 2000 clientes, con lo cual se evidencia que la estrategia actual de la PYMES debe ser enfocada en otra dirección para obtener un incremento en el número de clientes potenciales.

Cada una de las variables simuladas en la figura 2 son factibles de ser interpretadas. Por ejemplo, la variable ingreso denota que inicialmente su valor económico se ubica en los 25,000.00 dólares. No obstante, con el tiempo este valor económico disminuye hasta ubicarse en un valor de 5,000.00 dólares. Por lo tanto, sus políticas de gestión no son las adecuadas en este tiempo. Igualmente, se confirma que no son adecuadas las políticas que se utilizan al analizar la variable beneficios o utilidades, que en el mes cero tiene una pérdida inicial de 8,000.00 dólares, y que al transcurrir el tiempo se incrementa hasta obtener una pérdida de 10,000.00 dólares. Con estos resultados es evidente que la PYMES de servicios debe realizar cambios en su gestión empresarial si desea obtener rendimientos financieros positivos.

Por otro lado, al aplicar el modelo de gestión empresarial diseñado, es factible realizar un ejercicio similar en la PYMES de producción, lo cual se detalla a continuación: 


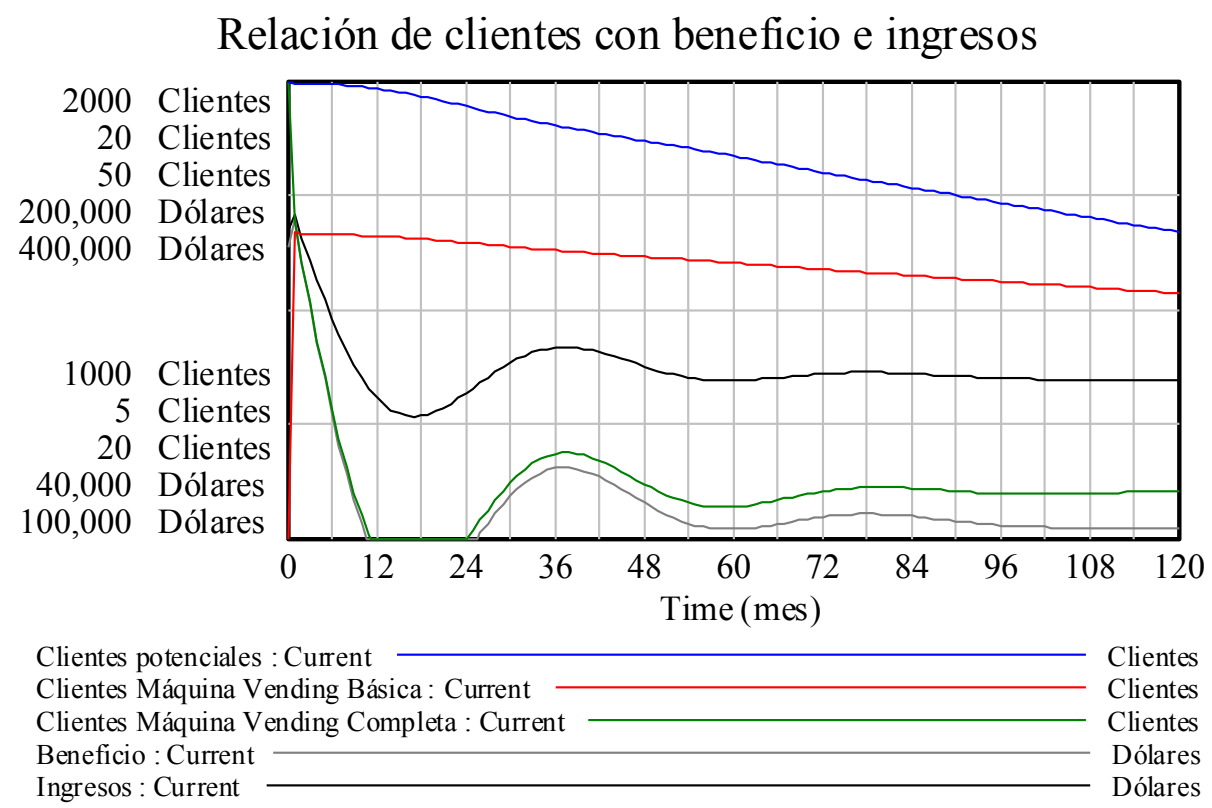

Figura 3. Simulación con base en variables clientes potenciales, clientes máquina vending básica, clientes máquina vending completa, ingresos y beneficio de la PYMES de producción (figura 1b)

En la figura 3, es posible analizar las variables auxiliares de beneficio e ingreso correspondientes a la empresa de producción, las cuales son positivas en su valor monetario, pero disminuyen considerablemente sin llegar a valores que denoten pérdida, ante lo cual es factible señalar que la PYMES debe actuar estratégicamente para que dichos valores en un momento determinado no lleguen a ser negativos.

De esta manera, la simulación efectuada a la empresa PYMES de producción, ratifica que el modelo de gestión empresarial diseñado para este tipo de empresas posibilita establecer políticas de gestión que permitan corregir o continuar por el camino actual en el que se encuentra la PYMES.

\section{CONCLUSIONES}

Los modelos de simulación basados en dinámica de sistemas son útiles para diversos trabajos, sean estos interdisciplinarios o educativos, por cuanto una de las facilidades de aplicar esta metodología es que los diversos actores pueden tener distintos grados de formalización en su pensamiento, facilitando así la comunicación a través de una forma concreta de representar y demostrar lo que se sabe y se necesita evidenciar.

El modelo diseñado explica razonablemente la influencia de los factores principales que afectan a las PYMES, por lo que el modelo desarrollado considera la influencia que tienen este tipo de empresas con el crecimiento o decrecimiento de la economía, además de incorporar variables que usualmente podrían ser difíciles de considerar en 
otro tipo de modelos, como es la calidad y la capacidad que la empresa dispone para atender a clientes. Así, el objetivo fundamental del trabajo fue validar el modelo diseñado, el mismo que fue posible hacerlo acorde a la técnica de recopilación de datos.

Los resultados que el modelo proporciona tienen relación con la calidad de la información de entrada, que si bien no es tan precisa como la requerida por un método de pronóstico cuantitativo, obligatoriamente debe ser coherente y consistente con la situación del contexto en estudio. Sin embargo, se debe señalar que los resultados alcanzados evidencian la sensibilidad de los datos recopilados, lo cual genera una confiabilidad expectante de ser considerados para no cometer errores en la administración de las PYMES y llevarlas al éxito. El modelo generado, además de posibilitar el pronóstico de resultados, permite la posibilidad cierta de análisis de escenarios, por cuanto la fortaleza de este permite integrar de manera didáctica, en un solo conjunto sistémico, conceptos y relaciones conocidas por la mayoría de personas inmersas en el mundo de las PYMES, pero a la vez desconocidas en el impacto que reflejan estas al analizarlas en conjunto.

Finalmente, se evidencia que el modelo diseñado, además de cumplir el objetivo de ser validado refleja que la dinámica de sistemas, sus simulaciones y escenarios de análisis, son una manera posible de anticiparse a la lógica administrativa que por varias ocasiones es utilizada, así como el aplicar estrategias específicas para las PYMES.

\section{REFERENCIAS}

Aguilar, C. (1997). Simulación de sistemas, aplicaciones en producción animal. Santiago: Pontificia Universidad Católica de Chile, Facultad de Agronomía.

Aracil, J. y Gordillo, F. (1997). Dinámica de Sistemas Madrid: Alianza.

Barrales, L., Peña, I. y Fernández, P. (2004). Validación de modelos: un enfoque aplicado. Agricultura Técnica, 64(1), 66-73. https://dx.doi.org/10.4067/S0365-28072004000100008. Accedido el 4 agosto 2017

Flavelle, P. (1992). A quantitative measure of model validation and its potential use for regulatory purpose. Adv. Water Resour., 15, 5-13. Disponible en http://www.scielo.cl/ scielo.php?script=sci_nlinks\&ref=274968\&pid=S03652807200400010000800004\&lng=es . Accedido el 22 de agosto 2017

Forrester, J. (1981). Dinámica industrial. El Ateneo: Buenos Aires.

Godoy, L. y Bartó, C. (2002). Validación y valoración de modelos en la dinámica de sistemas. Revista argentina de enseñanza de la ingeniería ,3(5), 31-47. http://www.ing.unrc.edu.ar/ raei/archivos/img/arc_2011-11-23_21_00_45-54.pdf. Accedido el 15 de agosto 2017

Góngora, G. y Madrid, A. (2010). El apoyo a la innovación de la PyME en México. Un estudio exploratorio. http: //redalyc.uaemex.mx./src/inicio/ArtPdf.jsp?!Cve=67413393004. Accedido el 10 de agosto 2017

Iñaki, S. (2010). Modelo de dinámica de sistemas para la implantación de tecnologías de la información en la gestión estratégica universitaria. [Tesis de doctorado sin publicar]. Universidad del País Vasco, San Sebastián.

Izquierdo, L., Galán J., Santos, J. y Del Olmo Martínez, R. (2008). Modelado de sistemas complejos mediante simulación basada en agentes y mediante dinámica de sistemas. Empiria. 
Revista De Metodología De Ciencias Sociales, O(16), 85-112, doi: http://dx.doi.org/10.5944/ empiria.16.2008.1391. Accedido 1 de agosto de 2017

Moss, S., Edmonds, B., Wallis, S. (1997). Validation and Verification of Computational. Models with Multiple Cognitive Agents. Centre for Policy Modelling Report, 97(25). Accedido el 8 de Agosto 2017

Putnam, H. (1972). Philosophy of Logic. New York: Harper and Row.

Samaniego, H. y Pascual, A. (2017). A business management model based on systems dynamics for small and medium enterprise. DYNA Management, 5(1). DOI: http://dx.doi.org/10.6036/ MN8358. Accedido el 29 de julio 2017

Samaniego, H. (2017). Desarrollo de un modelo de Gestión Empresarial para potencializar el crecimiento de la pequeña y mediana empresa (PYMES) en la región centro norte de Quito, Ecuador. [Tesis de doctorado sin publicar]. Universidad Internacional Iberoamericana. México. 